\title{
Classical dynamics on three-dimensional fuzzy space
}

\author{
FG Scholtz \\ National Institute for Theoretical Physics (NITheP), Stellenbosch 7602, South Africa \\ and Institute of Theoretical Physics, Stellenbosch University, Stellenbosch 7602, South Africa
}

(Received 14 October 2018; published 29 November 2018)

\begin{abstract}
We derive the path integral action for a particle moving in three-dimensional fuzzy space. From this we extract the classical equations of motion. These equations have rather surprising and unconventional features: they predict a cutoff in energy, a generally spatial-dependent limiting speed, orbital precession remarkably similar to the general-relativistic result, flat velocity curves below a length scale determined by the limiting velocity and included mass, displaced planar motion, and the existence of two dynamical branches of which only one reduces to Newtonian dynamics in the commutative limit. These features may provide a stringent observational test for this scenario of noncommutativity.
\end{abstract}

DOI: 10.1103/PhysRevD.98.104058

\section{INTRODUCTION}

The structure of space-time at short length scales and the emergence of space-time as we perceive it at long length scales are probably the most challenging problems facing modern physics [1]. These issues are also at the core of the struggle to combine gravity and quantum mechanics into a unified theory and probably also links closely with the observational challenges of dark matter and energy.

One of the difficulties facing our understanding of spacetime at short length scales is the lack of observational data that can be accessed at energies and length scales available to us, either through accelerators or astronomical observation. Mostly the short length scale structure of space-time manifests itself at very high energies and short length scales inaccessible to current observational techniques.

One scenario for space-time at short length scales is that of noncommutative space-time, which has received considerable attention in the past few decades. This was originally proposed by Snyder [2] in an attempt to avoid the ultraviolet infinities of field theories. The discovery of renormalization pushed these ideas to the background until more recently when they resurfaced in the search for a consistent theory of quantum gravity. The compelling arguments of Doplicher et al. [3] highlighted the need for a revised notion of space-time at short length scales and gave strong arguments in favor of a noncommutative geometry. Shortly thereafter it was also noted that noncommutative coordinates occurred quite naturally in certain

Published by the American Physical Society under the terms of the Creative Commons Attribution 4.0 International license. Further distribution of this work must maintain attribution to the author(s) and the published article's title, journal citation, and DOI. Funded by SCOAP ${ }^{3}$. string theories [4], generally perceived to be the best candidate for a theory of quantum gravity. This sparked renewed interest in noncommutative space-time and the formulation of quantum mechanics [5] and quantum field theories on such spaces [6].

Despite the developments above, the observational consequences of noncommutativity remain elusive due to the smallness of the effect, especially on the microscopic level. In Refs. [7-9] it was argued that noncommutativity can have observational consequences at the macroscopic scale for Fermi gases at very high densities and/or temperatures. Yet, again, if noncommutativity is assumed to manifest itself at the Planck scale, these densities and temperatures are outside our observational window.

One other possible manifestation of noncommutativity on the macroscopic level may be in the modification of classical dynamics and gravity. This has seemingly not yet been explored systematically, which is the motivation for the current paper that aims to fill this gap, at least in the case of a fuzzy-space scenario. Modified Newtonian dynamics (MOND) [10] and modified gravity [11] have of course been topics of research for many years. Yet, although the present paper contains elements of MOND, there are many technical differences. Furthermore, in contrast to the phenomenological approach of MOND, the modified dynamics derived here follow from first principles using the action of a particle moving in a noncommutative space. The latter, in turn, is also systematically derived from the Schrödinger equation.

As our aim here is to study the motion of macroscopic objects, including planetary and galactic motion, it is sufficient to limit ourselves to the nonrelativistic regime of low velocities. The starting point of our derivation will therefore be the nonrelativistic Schrödinger equation. There are, of course, effects arising in planetary motion that stem 
from a general-relativistic description. The prime example is precession of the perihelion of a planet's orbit such as Mercury. We show here that noncommutative dynamics gives rise to the same effect, albeit numerically somewhat different. In addition, another feature of relativity that seems to emerge quite naturally is that of a limiting speed.

This paper is organized as follows. In Sec. II we give a generic expression for the action of a particle on two- and three-dimensional noncommutative space. In Sec. III we specify to a two-dimensional noncommutative plane and derive the modified equations of motion. In Sec. IV we consider three-dimensional fuzzy space and derive the modified equations of motion. Due to its observational importance, we spend considerable time discussing the implications of this modified dynamics in a number of subsections. In Sec. V, we introduce a further generalization of the modified dynamics found in Sec. IV. In Sec. VI we discuss the core findings, their implications, and open issues. Finally, we close with a summary and conclusions in Sec. VII.

\section{THE ACTION ON NONCOMMUTATIVE SPACE}

In noncommutative space coordinates are no longer commuting and simultaneous eigenstates of position cannot be found. This eliminates the standard time-slicing procedure using position eigenstates and the subsequent representation of the transition amplitude as a functional integral over position. Instead, one needs to replace the eigenstates of position with minimum-uncertainty states, which also form an overcomplete set, commonly referred to as coherent states. The standard time-slicing procedure can then again be implemented to write a coherent-state pathintegral representation of the transition amplitude. In general, this representation reads [12]

$$
\left\langle\ell_{f}, t_{f} \mid \ell_{i}, t_{i}\right\rangle=\int_{\ell\left(t_{i}\right)=\ell_{i}}^{\ell\left(t_{f}\right)=\ell_{f}}[d \mu(\ell)] e^{\frac{i}{\hbar} S},
$$

with the path-integral action

$$
S=\int_{t_{i}}^{t_{f}} d t\left\langle\ell(t)\left|i \hbar \frac{\partial}{\partial t}-H\right| \ell(t)\right\rangle .
$$

Here $|\ell\rangle$ is a set of overcomplete coherent states, i.e.,

$$
\int d \mu(\ell)|\ell\rangle\langle\ell|=\mathbf{1}
$$

where 1 is the identity on the Hilbert space.

This is the strategy we employ here to derive the pathintegral action for a particle on noncommutative space. In the next section we derive the action and classical dynamics of a particle in the two-dimensional noncommutative plane.

\section{THE TWO-DIMENSIONAL NONCOMMUTATIVE PLANE}

To start, we briefly recall the formulation of quantum mechanics on two-dimensional noncommutative space [5]. In this case the coordinate algebra is given by

$$
[\hat{x}, \hat{y}]=i \theta,
$$

where $\theta$ is a constant with dimensions of length squared, which we can take without loss of generality to be positive, and $\hat{x}, \hat{y}$ are Hermitian operators.

To develop the quantum theory [5], one first introduces a representation of this coordinate algebra on some Hilbert space $\mathcal{H}_{c}$, referred to as classical configuration space. In the case at hand, one notes that $b=\frac{1}{\sqrt{2 \theta}}(\hat{x}+i \hat{y})$ and $b^{\dagger}=$ $\frac{1}{\sqrt{2 \theta}}(\hat{x}-i \hat{y})$ are standard creation and annihilation operators. The radius operator is $\hat{r}^{2}=\hat{x}^{2}+\hat{y}^{2}=\theta\left(b^{\dagger} b+1\right)$. It is then natural to choose for $\mathcal{H}_{c}$ the Fock space for one oscillator [5] since each value of the quantized radius appears exactly once in this representation and in this sense the two-dimensional plane is completely covered once.

The next step is to introduce the quantum Hilbert space, denoted by $\mathcal{H}_{q}$. This is the space of all Hilbert-Schmidt operators acting on $\mathcal{H}_{c}$ and that are generated by the noncommutative coordinates. We denote states in $\mathcal{H}_{c}$ by $|\cdot\rangle$ and states in $\mathcal{H}_{q}$ by $\left.\mid \cdot\right)$. The inner product on $\mathcal{H}_{q}$ is $(\phi \mid \psi)=\operatorname{tr}_{c}\left(\phi^{\dagger} \psi\right)$, where $\operatorname{tr}_{c}$ denotes the trace over $\mathcal{H}_{c}$. A general element of $\mathcal{H}_{q}$ thus has the form $\left.\mid a_{n, m}\right)=\sum_{n, m} a_{n, m}|n\rangle\langle m|$, with $\sum_{n, m}\left|a_{n, m}\right|^{2}<\infty$. Note that the states $\mid n, m)=|n\rangle\langle m|$ form a complete orthonormal basis in $\mathcal{H}_{q}$.

From here the construction of the quantum theory proceeds as normal: one introduces observables as selfadjoint operators acting on $\mathcal{H}_{q}$ and the standard probabilistic interpretation. To distinguish these from operators on $\mathcal{H}_{c}$, we denote them by capitals. The only generalization is that a position measurement must now be interpreted in the context of a weak measurement or a positive-valued measure. A detailed discussion of this can be found in Ref. [5], where it was also shown that standard commutative quantum mechanics is recovered in the limit $\theta \rightarrow 0$.

The most important observable for our current purposes is the Hamiltonian given by [5]

$$
H=\frac{\bar{P} P}{2 m}+V(\hat{R}), \quad V(\hat{R})^{\dagger}=V(\hat{R}) .
$$

Here the action of the momentum operators on a generic element $\psi$ of $\mathcal{H}_{q}$ is defined as

$$
\left.\left.P|\psi\rangle=\mid-i \hbar \sqrt{\frac{2}{\theta}}[b, \psi]\right), \quad \bar{P}|\psi\rangle=\mid i \sqrt{\frac{2}{\theta}} \hbar\left[b^{\dagger}, \psi\right]\right) .
$$


Similarly, the action of the position operators is defined as

$\hat{X}|\psi\rangle=\mid \hat{x} \psi), \quad \hat{Y}|\psi\rangle=\mid \hat{y} \psi), \quad \hat{R}^{2}=\hat{X}^{2}+\hat{Y}^{2}$.

Note that momentum involves left and right multiplication, while position only involves left multiplication.

Another useful observable is the angular momentum, which acts as follows:

$$
\left.L|\psi\rangle=\mid \hbar\left[b^{\dagger} b, \psi\right]\right) .
$$

If the potential is a function of $\hat{R}$ only, this operator commutes with the Hamiltonian and is a conserved quantity.

To find the coherent-state path-integral action is now straightforward. One first introduces an overcomplete set of minimum-uncertainty states on $\mathcal{H}_{c}$ and $\mathcal{H}_{q}$. For $\mathcal{H}_{c}$, they are the standard normalized Glauber coherent states,

$$
|z\rangle=e^{-|z|^{2} / 2} e^{z b^{\dagger}}|0\rangle, \quad \int \frac{d \bar{z} d z}{\pi}|z\rangle\langle z|=\mathbf{1}_{c}
$$

and represent the best approximation to a position eigenstate or point in the plane. In this sense $z$ must then be interpreted as dimensionless complex coordinates on the plane, as is clear from the expectation values $x=\langle z|\hat{x}| z\rangle=$ $\sqrt{2 \theta} \operatorname{Re} z$ and $y=\langle z|\hat{y}| z\rangle=\sqrt{2 \theta} \operatorname{Im} z$.

From this, the corresponding coherent states on $\mathcal{H}_{q}$ can be easily written down,

$$
\mid z, w)=|z\rangle\langle w|
$$

Noting that

$$
\left.\mid z, w)=e^{-\frac{1}{2}(\bar{z} z+\bar{w} w)} \sum_{n, m=0}^{\infty} \frac{z^{n} w^{m}}{\sqrt{n ! m !}} \mid n, m\right),
$$

we have

$$
\left.\int \frac{d \bar{z} d z d \bar{w} d w}{\pi^{2}} \mid z, w\right)\left(z, w\left|=\sum_{n, m=0}^{\infty}\right| n, m\right)\left(n, m \mid=\mathbf{1}_{q} .\right.
$$

Keeping in mind that the time-evolution operator acts on $\mathcal{H}_{q}$, the path-integral representation of the transition amplitude in the coherent-state representation (10) can be easily found from Eq. (2) and is given by

$$
S=\int_{t_{i}}^{t_{f}} d t\left(z(t), w(t)\left|i \hbar \frac{\partial}{\partial t}-H\right| z(t), w(t)\right) .
$$

A simple computation yields the explicit form

$$
S=\int_{t_{i}}^{t_{f}} d t\left[\frac{i \hbar}{2}(\bar{z} \dot{z}-\dot{\bar{z}} z+\dot{\bar{w}} w-\bar{w} \dot{w})-H(z, \bar{z}, w, \bar{w})\right],
$$

with
$H(z, \bar{z}, w, \bar{w})=\frac{\hbar^{2}}{m \theta}((\bar{z}-\bar{w})(z-w)+1)+\tilde{V}(R)$.

Here $\quad R=\bar{z} z \quad$ and $\quad \tilde{V}(R)=(z, w|V(\hat{R})| z, w)=$ $\operatorname{tr}_{c}(|w\rangle\langle z|V(\hat{R})| z\rangle\langle w|)=\langle z|V(\hat{R})| z\rangle$. Note that the function $\tilde{V}$ is different from $V$ as a normal ordering is required to replace $\hat{R}$ by its expectation value. The rest of the terms in the action are computed in a similar way, with the only point of care being the right-acting operators in the kinetic energy term. The constant that appears comes from the normal ordering of right-acting operators to compute the coherent-state expectation value.

A more restrictive set of coherent states in which $z=w$ can also be introduced. They satisfy an overcompleteness relation of the form

$$
\left.\int \frac{d \bar{z} d z}{\pi} \mid z, z\right) \star\left(z, z \mid=\mathbf{1}_{q},\right.
$$

where $\star$ denotes the Voros product. In Ref. [13] these states were used to derive the path-integral action for a particle in the noncommutative plane. To make contact with that result, we introduce a change of variables from $w$ and $z$ to $z$ and $v$ with $w=v+z$. This gives the action

$$
S=\int_{t_{i}}^{t_{f}} d t\left[i \hbar(\dot{\bar{z}} v-\bar{v} \dot{z}-\bar{v} \dot{v})-\frac{\hbar^{2}}{m \theta} \bar{v} v-\left(\tilde{V}(R)+\frac{\hbar^{2}}{m \theta}\right)\right] .
$$

Noting that this action is quadratic in $v$, the $v$ integration can be performed explicitly to yield

$$
S=\int_{t_{i}}^{t_{f}} d t\left[m \theta \dot{\bar{z}}\left(1+\frac{i m \theta}{\hbar} \partial_{t}\right)^{-1} \dot{z}-\left(\tilde{V}(R)+\frac{\hbar^{2}}{m \theta}\right)\right],
$$

in agreement with Ref. [13]. (Note that Ref. [13] contains a sign misprint in the factor before $\partial_{t}$.)

With the action in hand, we can give precise meaning to the notion of classical dynamics in the sense of a saddle point of the action. Returning to Eq. (14), we can easily derive the equations governing the classical dynamics:

$$
\begin{gathered}
i \hbar \dot{\bar{w}}+\frac{\hbar^{2}}{m \theta}(\bar{z}-\bar{w})=0, \\
-i \hbar \dot{w}+\frac{\hbar^{2}}{m \theta}(z-w)=0, \\
-i \hbar \dot{\bar{z}}-\frac{\hbar^{2}}{m \theta}(\bar{z}-\bar{w})-\frac{\partial \tilde{V}}{\partial z}=0, \\
i \hbar \dot{z}-\frac{\hbar^{2}}{m \theta}(z-w)-\frac{\partial \tilde{V}}{\partial \bar{z}}=0 .
\end{gathered}
$$

Note that these equations still involve $\hbar$. In fact, the order of the limits $\hbar \rightarrow 0$ and $\theta \rightarrow 0$ is important here. Taking the 
$\theta \rightarrow 0$ limit first and then $\hbar \rightarrow 0$ gives a well-defined result, while the other order does not. In the former, one of course expects (and indeed gets) the classical commutative result.

Assuming that the potential only depends on $R$, there are two constants of motion related to a $U(1)$ symmetry involving a global phase change on all variables and time-translation invariance: the angular momentum and energy,

$$
L=\hbar(\bar{z} z-\bar{w} w), \quad E=H(z, \bar{z}, w, \bar{w}) .
$$

Using the equations of motion (19), one can check explicitly that these quantities are indeed conserved.

We are not interested in the dynamics of $w$, but only the physical coordinates $z$ and would like to eliminate the former. As the two last equations of Eq. (19) are algebraic equations for $w$ and $\bar{w}$, we can solve for them and compute the equation of motion for $z$ by substituting in the first two equations of Eq. (19). This yields

$$
\begin{aligned}
& \ddot{z}=-\frac{1}{m \theta} \frac{\partial \tilde{V}}{\partial \bar{z}}-\frac{i}{\hbar} \frac{d}{d t}\left(\frac{\partial \tilde{V}}{\partial \bar{z}}\right), \\
& \ddot{\bar{z}}=-\frac{1}{m \theta} \frac{\partial \tilde{V}}{\partial z}+\frac{i}{\hbar} \frac{d}{d t}\left(\frac{\partial \tilde{V}}{\partial z}\right) .
\end{aligned}
$$

We can return to the dimensionful coordinates $x$ and $y$ by writing

$$
z=\frac{1}{2 \theta}(x+i y), \quad \bar{z}=\frac{1}{2 \theta}(x-i y),
$$

and

$$
\frac{\partial}{\partial z}=\sqrt{\frac{\theta}{2}}\left(\frac{\partial}{\partial x}-i \frac{\partial}{\partial y}\right), \quad \frac{\partial}{\partial \bar{z}}=\sqrt{\frac{\theta}{2}}\left(\frac{\partial}{\partial x}+i \frac{\partial}{\partial y}\right) .
$$

This gives the equations of motion

$$
\begin{aligned}
& \ddot{x}=-\frac{1}{m} \frac{\partial V}{\partial x}+\frac{\theta}{\hbar} \frac{d}{d t}\left(\frac{\partial V}{\partial y}\right), \\
& \ddot{y}=-\frac{1}{m} \frac{\partial V}{\partial y}-\frac{\theta}{\hbar} \frac{d}{d t}\left(\frac{\partial V}{\partial x}\right) .
\end{aligned}
$$

These are the standard Newtonian equations of motion, supplemented by a noncommutative correction. In the $\theta \rightarrow 0$ limit, we recover standard Newtonian dynamics. As already mentioned, the limit $\hbar \rightarrow 0$ cannot be taken before the commutative limit.

We do not explore the consequences of this modified dynamics here, but rather postpone the in-depth analysis to the three-dimensional case, which is much more interesting and physically relevant.

\section{THREE-DIMENSIONAL FUZZY SPACE}

In this section we study the modified classical dynamics on three-dimensional fuzzy space. The noncommutative quantum mechanics on three-dimensional fuzzy space has been studied extensively in Refs. [8,14-16]. In these studies it was shown that this formulation reduces to commutative quantum mechanics in the commutative limit and that it is a realistic description of the physics at low energies. At high energies there are strong deviations from commutative quantum mechanics, most notably the existence of an upper bound on the energy of a free particle $[14,16]$, given by $E_{\max }=\frac{2 \hbar^{2}}{m \lambda^{2}}$, and a finite density of singleparticle states [9]. Our interest here is to see how this translates into the classical dynamics and what observational consequences it may have.

We start by reviewing the formulation of noncommutative quantum mechanics on three-dimensional fuzzy space, which follows essentially the same logic as for the twodimensional noncommutative plane. The main difference is the modification of the coordinate algebra as the commutation relations adopted in the case of the noncommutative plane break rotational symmetry. To rectify this, we adopt fuzzy-sphere commutation relations,

$$
\left[\hat{x}_{i}, \hat{x}_{j}\right]=2 i \lambda \varepsilon_{i j k} \hat{x}_{k} .
$$

Here $\lambda$ has units of length and $\varepsilon_{i j k}$ is the standard completely antisymmetric tensor.

The representation we choose for this coordinate algebra is the standard Schwinger realization of $S U(2)$. Thus, classical configuration space $\mathcal{H}_{c}$ is a two-boson-mode Fock space on which the coordinates are realized as

$$
\hat{x}_{i}=\lambda a_{\alpha}^{\dagger} \sigma_{\alpha \beta}^{(i)} a_{\beta} .
$$

Here a summation over repeated indices is implied, $\alpha$, $\beta=1,2, \sigma_{\alpha \beta}^{(i)}, i=1,2,3$ are the Pauli spin matrices, and $a_{\alpha}^{\dagger}$ and $a_{\alpha}$ are standard boson creation and annihilation operators. The radius operator is

$$
\hat{r}^{2}=\hat{x}_{i} \hat{x}_{i}=\lambda^{2} \hat{n}(\hat{n}+2),
$$

where $\hat{n}=a_{\alpha}^{\dagger} a_{\alpha}$ is the boson-number operator. Note that the radius operator is also the Casimir of $S U(2)$ and commutes with the coordinates. As a measure of the radius, we use

$$
\hat{r}=\lambda(\hat{n}+1),
$$

which is to leading order in $\lambda$ the square root of $\hat{r}^{2}$. Note that this representation contains each $S U(2)$ representationand thus each quantized radius - exactly once, and therefore again corresponds to a complete single covering of $R^{3}$, commonly referred to as fuzzy space. 
The quantum Hilbert space $\mathcal{H}_{q}$ is now defined as the algebra of operators generated by the coordinates, i.e., the operators acting on $\mathcal{H}_{c}$ that commute with $\hat{r}^{2}$ and have a finite norm with respect to a weighted Hilbert-Schmidt inner product [16]:

$$
\mathcal{H}_{q}=\left\{\psi=\sum_{m_{i}, n_{i}=0}^{\infty} C_{n_{1}, n_{2}}^{m_{1}, m_{2}}\left(a_{1}^{\dagger}\right)^{m_{1}}\left(a_{2}^{\dagger}\right)^{m_{2}} a_{1}^{n_{1}} a_{2}^{n_{2}}: m_{1}+m_{2}=n_{1}+n_{2} \quad \text { and } \quad \operatorname{tr}_{\mathfrak{c}}\left(\psi^{\dagger} \hat{r} \psi\right)<\infty\right\} \text {. }
$$

The inner product on $\mathcal{H}_{q}$ is

$$
(\psi \mid \phi)=4 \pi \lambda^{2} \operatorname{tr}_{c}\left(\psi^{\dagger} \hat{r} \phi\right)=4 \pi \lambda^{3} \operatorname{tr}_{c}\left(\psi^{\dagger}(\hat{n}+1) \phi\right),
$$

with the trace taken over $\mathcal{H}_{c}$. This choice of the inner product is motivated by the observation that the norm of the operator that projects onto the subspace of spheres with radius $r \leq \lambda(N+1)$, with $N$ large, corresponds to the volume of a sphere in three-dimensional Euclidean space [16].

We use the standard $|\cdot\rangle$ notation for elements of $\mathcal{H}_{c}$ and |.) for elements of $\mathcal{H}_{q}$. It is important to note here that, in contrast to the two-dimensional noncommutative plane, the quantum Hilbert space is here restricted to only those operators on $\mathcal{H}_{c}$ that commute with the Casimir operator. This will be an important restriction in what follows.

Quantum observables are identified with self-adjoint operators acting on $\mathcal{H}_{q}$. We again use capitals to distinguish them from operators acting on $\mathcal{H}_{c}$. These include the coordinates which act through left multiplication as

$$
\left.\left.\hat{X}_{i} \mid \psi\right)=\mid \hat{x}_{i} \psi\right)
$$

and the angular momentum operators which act adjointly according to

$\left.\left.\hat{L}_{i} \mid \psi\right)=\mid \frac{\hbar}{2 \lambda}\left[\hat{x}_{i}, \psi\right]\right), \quad$ with $\quad\left[\hat{L}_{i}, \hat{L}_{j}\right]=i \hbar \varepsilon_{i j k} \hat{L}_{k}$.

The noncommutative analogue of the Laplacian is defined as

$\left.\left.\hat{\Delta}|\psi\rangle=-\mid \frac{1}{\lambda \hat{r}}\left[\hat{a}_{\alpha}^{\dagger},\left[\hat{a}_{\alpha}, \psi\right]\right]\right)=\mid \frac{1}{\lambda^{2}(\hat{n}+1)}\left[\hat{a}_{\alpha}^{\dagger},\left[\hat{a}_{\alpha}, \psi\right]\right]\right)$

and can be shown to commute with the three angular momentum operators [16].

The Hamiltonian is given by

$$
\hat{H}=-\frac{\hbar^{2}}{2 m} \hat{\Delta}+V(\hat{R}),
$$

where $\hat{R}$ is the radius operator that acts as

$$
\hat{R} \mid \psi)=\mid \lambda(\hat{n}+1) \psi), \quad \hat{n}=a_{\alpha}^{\dagger} a_{\alpha} .
$$

From the discussion above it should be clear that the angular momentum operators commute with the Hamiltonian and are therefore conserved. There is a further important conserved quantity, namely, the operator $\hat{\Gamma}$, which acts as follows:

$$
\left.\hat{\Gamma}|\psi\rangle=\mid\left[a_{\alpha}^{\dagger} a_{\alpha}, \psi\right]\right) .
$$

It is simple to check explicitly that it does in fact commute with the Hamiltonian.

To facilitate the construction of the classical dynamics on fuzzy space, we enlarge the quantum Hilbert space $\mathcal{H}_{q}$ to include all Hilbert-Schmidt operators acting on $\mathcal{H}_{c}$, i.e., all operators with finite norm generated by the creation and annihilation operators $a_{\alpha}^{\dagger}$ and $a_{\alpha}$. The inner product is still given by Eq. (34). We denote this enlarged space by $\mathcal{H}_{q}^{0}$. Clearly, $\mathcal{H}_{q} \subset \mathcal{H}_{q}^{0}$. From the definition of $\mathcal{H}_{q}$, it is then clear that physical states, i.e., states that belong to the subspace $\mathcal{H}_{q}$ must satisfy the constraint

$$
\hat{\Gamma} \mid \psi)=0
$$

Note that since $\hat{\Gamma}$ is conserved, initial states that satisfy this condition will do so at all times. Below we use this property explicitly in the construction of the path-integral representation of physical transition amplitudes.

We now proceed with the construction of the pathintegral representation of physical transition amplitudes. The first step is to get rid of the weighted inner product in Eq. (34). This can be done by redefining the wave functions as follows:

$$
\tilde{\psi}=\sqrt{\hat{r}} \psi
$$

The inner product then assumes the standard form

$$
(\tilde{\psi} \mid \tilde{\phi})=4 \pi \lambda^{2} \operatorname{tr}_{c}\left(\tilde{\psi}^{\dagger} \tilde{\phi}\right) .
$$

However, upon doing this we must also transform the Hamiltonian, or any other observable, as follows:

$$
\hat{\tilde{H}}=\sqrt{\hat{r}} H \frac{1}{\sqrt{\hat{r}}} .
$$


From here on we work with this quantum Hilbert space in which the inner product is given by Eq. (43) and observables are transformed as in Eq. (44). We denote this space by $\tilde{\mathcal{H}}_{q}$ and its enlargement by $\tilde{\mathcal{H}}_{q}^{0}$. Note that the constants of motion $\hat{\Gamma}$ and $\hat{L}_{i}$ are unchanged by this transformation.

It is obvious that $\hat{\Gamma}$ and $\hat{L}_{i}$ also commute with $\hat{\tilde{H}}$ and are conserved under the time evolution generated by this Hamiltonian. It is also clear that physical states are still characterized by the constraint

$$
\hat{\Gamma} \mid \tilde{\psi})=0 .
$$

We introduce the standard minimum-uncertainty states on $\mathcal{H}_{c}$ as Glauber coherent states, which form an overcomplete basis

$\left|z_{\alpha}\right\rangle=e^{-\mid \bar{z}_{\alpha} z_{\alpha} / 2} e^{z_{\alpha} a_{\alpha}^{\dagger}}|0\rangle, \quad \int \frac{d \bar{z}_{\alpha} d z_{\alpha}}{\pi^{2}}\left|z_{\alpha}\right\rangle\left\langle z_{\alpha}\right|=\mathbf{1}_{c}$.

The dimensionful physical coordinates are now identified as

$$
x_{i}=\left\langle z_{\alpha}\left|\hat{x}_{i}\right| z_{\alpha}\right\rangle=\lambda \bar{z}_{\alpha} \sigma_{\alpha \beta}^{(i)} z_{\beta} .
$$

As in the two-dimensional noncommutative plane, we can correspondingly introduce coherent states on $\tilde{\mathcal{H}}_{q}^{0}$ as

$$
\left.\mid z_{\alpha}, w_{\alpha}\right)=\left|z_{\alpha}\right\rangle\left\langle w_{\alpha}\right|
$$

They are overcomplete and

$$
\left.\int \frac{d \bar{z}_{\alpha} d z_{\alpha} d \bar{w}_{\alpha} d w_{\alpha}}{\pi^{4}} \mid z_{\alpha}, z_{\alpha}\right)\left(z_{\alpha}, w_{\alpha} \mid=\tilde{\mathbf{1}}_{\mathbf{q}}^{\mathbf{0}} .\right.
$$

It is interesting to note the close relation between these states and the "string states" introduced in Ref. [17]. It is also important to note that the states $\left.\mid z_{\alpha}, w_{\alpha}\right)$ are not all physical. However, we are interested in physical transition amplitudes, which implies that if the initial state is physical, all of the states at intermediate times are also physical as $\hat{\Gamma}$ commutes with $\hat{\tilde{H}}$. As the states $\left.\mid z_{\alpha}, w_{\alpha}\right)$ resolve the identity on $\tilde{\mathcal{H}}_{q}^{0}$, we can safely use them to insert the identity at intermediate times into a time-slicing procedure, provided that the initial state is physical. Indeed, if this is done, the constraint must appear as a conserved quantity in the resulting action and we must simply require it to vanish to satisfy the condition of physicality of the initial state.

Following this approach, the general result of Eq. (2) is still applicable and to obtain the path-integral action we therefore only have to compute the action

$$
S=\int_{t_{i}}^{t_{f}} d t\left(z_{\alpha}(t), w_{\alpha}(t)\left|i \hbar \frac{\partial}{\partial t}-\hat{\tilde{H}}\right| z_{\alpha}(t), w_{\alpha}(t)\right) .
$$

To simplify matters, it is convenient to introduce dimensionless quantities from here on, which we denote by capital letters. We introduce the following time scale $t_{0}$, energy scale $e_{0}$, dimensionless time $T$, dimensionless coordinates $X_{i}$, and dimensionless energy $E$ :

$t_{0}=\frac{m \lambda^{2}}{\hbar}, \quad e_{0}=\frac{\hbar}{t_{0}}, \quad T=\frac{t}{t_{0}}, \quad X_{i}=\frac{x_{i}}{\lambda}, \quad E=\frac{e}{e_{0}}$.

The dimensionless action $\tilde{S}=\frac{S}{\hbar}$ can than be explicitly computed. The computation is slightly more involved than in the case of the two-dimensional noncommutative plane, but still straightforward. We find

$$
\begin{aligned}
\tilde{S}= & \int_{T_{i}}^{T_{f}} d T\left[\frac{i}{2}\left(\bar{z}_{\alpha} \dot{z}_{\alpha}-\dot{\bar{z}}_{\alpha} z_{\alpha}+\dot{\bar{w}}_{\alpha} w_{\alpha}-\bar{w}_{\alpha} \dot{w}_{\alpha}\right)\right. \\
& \left.-\tilde{H}\left(z_{\alpha}, \bar{z}_{\alpha}, w_{\alpha}, \bar{w}_{\alpha}\right)\right],
\end{aligned}
$$

where

$$
\begin{aligned}
\tilde{H}(z, \bar{z}, w, \bar{w})= & \left(f_{1}(R) \bar{z}_{\alpha} z_{\alpha}-f_{2}(R)\left(\bar{z}_{\alpha} w_{\alpha}+z_{\alpha} \bar{w}_{\alpha}\right)\right. \\
& \left.+f_{3}(R) \bar{w}_{\alpha} w_{\alpha}\right)+W(R) .
\end{aligned}
$$

Here,

$$
\begin{gathered}
R=\bar{z}_{\alpha} z_{\alpha} \\
f_{1}(R)=\frac{1}{2}\left\langle z_{\alpha}\left|\frac{1}{\hat{n}+2}\right| z_{\alpha}\right\rangle \\
f_{2}(R)=\frac{1}{2}\left\langle z_{\alpha}\left|\frac{1}{\sqrt{(\hat{n}+1)(\hat{n}+2)}}\right| z_{\alpha}\right\rangle \\
f_{3}(R)=\frac{1}{2}\left\langle z_{\alpha}\left|\frac{1}{\hat{n}+1}\right| z_{\alpha}\right\rangle \\
W(R)=\frac{1}{e_{0}}\left\langle z_{\alpha}|V(\hat{R})| z_{\alpha}\right\rangle+2 f_{3}(R) \equiv \tilde{V}(R)+2 f_{3}(R) .
\end{gathered}
$$

Note that $R$, all of the $f_{i}(R)$, and $W(R)$ are dimensionless.

The equations of motion determining the classical dynamics can now be easily derived and are given by

$$
\begin{gathered}
\dot{z}_{\alpha}=-i \frac{\partial \tilde{H}}{\partial \bar{z}_{\alpha}}, \\
\dot{\bar{z}}_{\alpha}=i \frac{\partial \tilde{H}}{\partial z_{\alpha}}, \\
\dot{w}_{\alpha}=i \frac{\partial \tilde{H}}{\partial \bar{w}_{\alpha}}, \\
\dot{\bar{w}}_{\alpha}=-i \frac{\partial \tilde{H}}{\partial \bar{w}_{\alpha}} .
\end{gathered}
$$


There are five conserved quantities: four are related to a $U(2)$ symmetry, and the fifth is a conserved energy related to time-translation invariance. These are easily found to be

$$
\begin{aligned}
\Gamma & =\bar{z}_{\alpha} z_{\alpha}-\bar{w}_{\alpha} w_{\alpha}, \\
L_{i} & =\bar{z}_{\alpha} \sigma_{\alpha \beta}^{(i)} z_{\beta}-\bar{w}_{\alpha} \sigma_{\alpha \beta}^{(i)} w_{\beta}, \\
E & =\tilde{H}(z, \bar{z}, w, \bar{w}) .
\end{aligned}
$$

It can be checked directly from Eqs. (59)-(62) that these quantities are indeed constant in time. The first, $\Gamma$, is simply the expectation value of the conserved quantity $\hat{\Gamma}$ in the state $\left.\mid z_{\alpha}, w_{\alpha}\right)$ and therefore is naturally conserved. This quantity also determines whether states are physical or not and must vanish for physical states. We must therefore require $\Gamma=0$. The $L_{i}$ are just the expectation values of the momentum operators $\hat{L}_{i}$ in the same state and therefore are also conserved. Finally, $\tilde{H}(z, \bar{z}, w, \bar{w})$ is just the Hamiltonian which - as it is not explicitly time dependent-is conserved.

Our interest is not in the equations of motion of the $z_{\alpha}$ and $w_{\alpha}$, but rather in the equations of motion of the physical, dimensionless coordinates $X_{i}=\frac{x_{i}}{\lambda}$, with the $x_{i}$ given in Eq. (47). We must therefore eliminate $z_{\alpha}$ and $w_{\alpha}$ in favor of these. This is a long and tedious calculation that can fortunately be done efficiently with MATHEMATICA. The easiest way to proceed is to first parametrize the $z_{\alpha}$ as follows:

$$
\begin{aligned}
& z_{1}=\sqrt{R} \cos \left(\frac{\theta}{2}\right) e^{-i \frac{\phi}{2}} e^{i \gamma} \\
& z_{2}=\sqrt{R} \sin \left(\frac{\theta}{2}\right) e^{i \frac{\phi}{2}} e^{i \gamma},
\end{aligned}
$$

and the corresponding complex conjugates where $R>0$, and $\theta, \phi$ and $\gamma$ are real. With this parametrization the coordinates take the standard form in spherical coordinates,

$$
\begin{aligned}
& X_{1}=R \sin \theta \cos \phi, \\
& X_{2}=R \sin \theta \sin \phi, \\
& X_{3}=R \cos \theta .
\end{aligned}
$$

Note that the global phase $\gamma$ drops out of these expressions, but not from the time derivatives.

One now proceeds as follows: solve for the $w_{\alpha}$ from the algebraic equations (59)-(60) in terms of the $z_{\alpha}$ and their time derivatives (these expressions also contain $\dot{\gamma}$ ); solve $\dot{\gamma}$ from the constraint $\Gamma=0$; substitute this back into the expressions for the second-order time derivatives of the coordinates, computed using the equations of motion (59)-(62). Although the intermediate steps are involved, the final result is fairly simple and reads as follows:

$$
\begin{aligned}
\ddot{\vec{X}}_{ \pm}= & a_{ \pm}(R, V) \vec{X}+b_{ \pm}(R, V)(\vec{X} \times \dot{\vec{X}}) \\
& +c_{ \pm}(R)((\vec{X} \times \dot{\vec{X}}) \times \dot{\vec{X}}) .
\end{aligned}
$$

Here, $R^{2}=\vec{X} \cdot \vec{X}$ and

$$
\begin{aligned}
a_{ \pm}(R, V) & =4 R^{2} f_{2}(R)^{2} g_{1}(R) \pm \frac{g_{2}^{\prime}(R)}{R} \sqrt{4 R^{2} f_{2}(R)^{2}-\dot{\vec{X}} \cdot \dot{\vec{X}}} \\
b_{ \pm}(R, V) & =\frac{g_{2}^{\prime}(R)}{R} \pm g_{1}(R) \sqrt{4 R^{2} f_{2}(R)^{2}-\dot{\vec{X}} \cdot \dot{\vec{X}}} \\
c_{ \pm}(R) & =g_{1}(R) .
\end{aligned}
$$

Here,

$$
\begin{aligned}
& g_{1}(R)=\frac{1}{R^{2}}+\frac{f_{2}^{\prime}(R)}{f_{2}(R) R}, \\
& g_{2}(R)=R\left(f_{1}(R)+f_{3}(R)\right)+W(R),
\end{aligned}
$$

and the prime denotes a derivative with respect to $R$.

The dimensionless conserved quantities can also be computed, but now there are only four as the constraint $\Gamma=0$ is satisfied by construction. They are

$$
\begin{aligned}
\vec{L}_{ \pm}= & \frac{1}{4 f_{2}(R)^{2} R^{2}} \\
& \times\left[\sqrt{4 R^{2} f_{2}(R)^{2}-\dot{\vec{X}} \cdot \dot{\vec{X}}}(\vec{X} \times \dot{\vec{X}}) \pm(\vec{X} \times \dot{\vec{X}}) \times \dot{\vec{X}}\right],
\end{aligned}
$$

$$
E_{ \pm}=g_{2}(R) \pm \sqrt{4 R^{2} f_{2}(R)^{2}-\dot{\vec{X}} \cdot \dot{\vec{X}}}
$$

Note that there are two branches, denoted by \pm . Indeed, it is clear from Eq. (70) that the branch is determined by the sign of $E-g_{2}(R)$. The use of two branches in the equations of motion is inconvenient, but it turns out that a unified treatment is possible when one considers the radial motion in terms of an effective potential. We return to this in the next section.

One can benchmark these results in a number of ways. First, one can check, using the equations of motion (66), that the constants of motion are indeed constant in time, which turns out to be the case. Second, one can solve the equations of motion (59)-(62) numerically and check that this also solves Eq. (66). This also checks out. In this process one also finds that both branches are needed to describe the full dynamics. We discuss these equations of motion in more detail in the next section.

This is the most general form of the equations of motion. Indeed, in this form one may view the $f_{i}(R)$ as arbitrary functions, but note that if this is done there is a redundancy in $f_{1}(R), f_{3}(R)$, and $W(R)$ as only the combination of $g_{2}(R)$ plays a role. Since this turns out to be a useful point of view, we explore it further in Sec. V. For our current purposes, though, we continue to compute the functions $f_{i}(R)$ as they appear in Eqs. (55)-(57). 
To do this, we note that the coherent state (summation over repeated indices is implied) $\left|z_{\alpha}\right\rangle=e^{-\frac{\bar{z} \alpha z \alpha}{2}} e^{z_{\beta} a_{\beta}^{\dagger}}|0\rangle$ can be rewritten, upon introducing a new creation operator $A^{\dagger}=\frac{1}{\sqrt{R}} z_{\beta} a_{\beta}^{\dagger}\left(R=\bar{z}_{\alpha} z_{\alpha}\right)$, as $\left|z_{\alpha}\right\rangle=e^{-\frac{R}{2}} e^{R A^{\dagger}}|0\rangle$. It then follows easily that for any function $g(\hat{n}+1)$

$$
g(R) \equiv\left\langle z_{\alpha}|g(\hat{n}+1)| z_{\alpha}\right\rangle=e^{-R} \sum_{n=0}^{\infty} g(n+1) \frac{R^{n}}{n !} .
$$

From this we also easily deduce the general relation

$$
\left\langle z_{\alpha}|g(\hat{n}+2)| z_{\alpha}\right\rangle=g(R)+\frac{d g(R)}{d R} .
$$

Similar relations can be derived for $g(\hat{n}+k)$, for a positive integer $k$, by iterating Eq. (72).

By explicit summation, we can now easily compute $f_{3}(R)$ exactly. Using Eq. (72), we can extract $f_{1}(R)$ exactly. Finally, upon noting that

$$
\left\langle z_{\alpha}\left|\frac{1}{(\hat{n}+1)^{k}}\right| z_{\alpha}\right\rangle=e^{-R} \sum_{n=0}^{\infty} \frac{1}{(n+1)^{k}} \frac{R^{n}}{n !} \sim \frac{1}{R^{k}}
$$

for large $R$, we can extract the large- $R$ behavior of $f_{2}$ through an expansion in orders of $\frac{1}{\hat{n}+1}$. The final result is

$$
\begin{aligned}
& f_{1}(R)=\frac{1}{2 R}-\frac{1-e^{-R}}{2 R^{2}} \approx \frac{1}{2 R}-\frac{1}{2 R^{2}}, \\
& f_{2}(R) \approx \frac{1}{2 R}-\frac{1}{4 R^{2}}-\frac{1}{16 R^{3}}, \\
& f_{3}(R)=\frac{1-e^{-R}}{2 R} \approx \frac{1}{2 R} .
\end{aligned}
$$

When one is interested in long length scales, it is sufficient to approximate these functions by

$$
f_{i}(R)=\frac{1}{2 R}, \quad \forall i
$$

In the lowest-order approximation (75) the equations and constant of motion simplify considerably and provide a useful benchmark for understanding the dynamics. Let us therefore consider this approximation. Substituting Eq. (75) into Eqs. (66) and (67) yields

$$
\ddot{\vec{X}}_{ \pm}=\frac{W^{\prime}(R)}{R}[(\vec{X} \times \dot{\vec{X}}) \pm \sqrt{1-\dot{\vec{X}} \cdot \dot{\vec{X}}} \vec{X}] .
$$

The dimensionless conserved quantities are

$$
\begin{gathered}
\vec{L}_{ \pm}=\sqrt{1-\dot{\vec{X}} \cdot \dot{\vec{X}}}(\vec{X} \times \dot{\vec{X}}) \pm((\vec{X} \times \dot{\vec{X}}) \times \dot{\vec{X}}), \\
E_{ \pm}=1 \pm \sqrt{1-\dot{\vec{X}} \cdot \dot{\vec{X}}}+W(R) .
\end{gathered}
$$

Equations (66) and (76) have rather interesting consequences as they suggest that the dimensionless speed $V^{2}=$ $\dot{\vec{X}} \cdot \dot{\vec{X}}$ of a projectile is limited in a generally spatialdependent way determined by the function $f_{2}(R)$. At long length scales $(R \gg 1)$ it becomes spatially independent and $V^{2} \leq 1$. Related to this, the dimensionless kinetic energy $E_{k}=1 \pm \sqrt{1-\dot{\vec{X}} \cdot \dot{\vec{X}}}$ is bounded by $E_{k} \leq 2$. Note that energies $E_{k}>1$ are described by the plus branch. This bound on the energy is in complete agreement with the bound found on the quantum level $[14,16]$. The bound on the speed of an object comes as a surprise and closer scrutiny traces it back to the condition of physicality (41) of the wave functions. More insight can be obtained by considering the dimensionful form of the equations of motion (76),

$$
\ddot{\vec{x}}_{ \pm}=\frac{w^{\prime}(r)}{m r}\left[\frac{m \lambda}{\hbar}(\vec{x} \times \dot{\vec{x}}) \pm \sqrt{1-\left(\frac{m \lambda}{\hbar}\right)^{2} \dot{\vec{x}} \cdot \dot{\vec{x}} \vec{x}}\right],
$$

and the conserved quantities

$$
\begin{aligned}
& \vec{\ell}_{ \pm}= \hbar \vec{L} \\
&= m\left[\sqrt{1-\left(\frac{m \lambda}{\hbar}\right)^{2} \dot{\vec{x}} \cdot \dot{\vec{x}}}(\vec{x} \times \overrightarrow{\dot{x}}) \pm \frac{m \lambda}{\hbar}((\vec{x} \times \dot{\vec{x}}) \times \dot{\vec{x}})\right] \\
& e_{ \pm}=\frac{\hbar^{2}}{m \lambda^{2}}\left[1 \pm \sqrt{1-\left(\frac{m \lambda}{\hbar}\right)^{2} \dot{\vec{x}} \cdot \dot{\vec{x}}}\right]+w(r)
\end{aligned}
$$

Here $w(r)=V(r)+\frac{2 \hbar^{2} f_{3}(r / \lambda)}{m \lambda^{2}}$, where $V(r)$ is the dimensionful potential. In this form one recognizes the bound on the speed of a projectile as $v_{0}=\frac{\hbar}{m \lambda}$. Also note that upon restoration of dimensions, the dimensionful length scale at which the limiting speed becomes spatially independent is the noncommutative parameter $\lambda$. As this is presumably a very short scale, the limiting speed can essentially be viewed as spatially independent. However, if the function $f_{2}(R)$ is treated more generally as described in Sec. V, this may not be the case. We postpone further discussion of this result to Sec. VI after we have established some other results that have a bearing on this.

Finally, note that, apart from a singular correction in the potential $w$, which vanishes in the $\hbar \rightarrow 0$ limit, one recovers the standard Newtonian equations in the $\lambda \rightarrow 0$ limit for the minus branch. This again emphasizes that the commutative and classical limits have to be taken with great care, as the order matters. In fact, here it is not possible to take either of these limits without encountering a singularity: the only sensible limit seems to be one in which the ratio between $\hbar$ and $\lambda$ is kept fixed. In Sec. VI, we 
argue that this is in fact also necessary from other physical considerations.

\section{A. General properties of orbitals}

The first step in understanding the motion implied by Eqs. (66) and (76) is to understand the relationship between the different conserved quantities, the velocities, and acceleration. For simplicity, we consider the dimensionless quantities. It is straightforward to establish the following relations that hold on both branches and for the general equation of motion (66) and their long-scale approximation (76):

$$
\begin{gathered}
\vec{L}_{ \pm} \cdot \dot{\vec{X}}=0, \\
\vec{L}_{ \pm} \cdot \ddot{\vec{X}}_{ \pm}=0, \\
\vec{L}_{ \pm} \cdot \vec{X}=\mp \vec{L} \cdot \vec{L} \equiv \mp L^{2} .
\end{gathered}
$$

We note that $\vec{L} \cdot \vec{X}$ is conserved in time. Note that this result contrasts with standard central-potential motion, for which $\vec{L} \cdot \vec{X}=0$. The motion is, however, still planar as in the case of a standard central potential, but the plane is displaced along the direction of $\vec{L}$, leading to $\vec{L} \cdot \vec{X} \neq 0$. Specifically, in the case of gravity this implies that the mass creating the gravitational force no longer lies in the plane of motion.

Another important point to note is that the conserved quantity $\vec{X} \cdot \vec{L}$ switches signs between the two branches. This means that the dynamics of the two branches do not mix, except in the case when $L=0$. The minus branch reduces to standard Newtonian dynamics in the commutative limit and has a kinetic energy $E_{k}<1$. Note that this also brings about an asymmetry: the plane of motion is always displaced in the direction of $\vec{L}$ for the minus branch and oppositely for the plus branch.
Finally, it is convenient to introduce the vector $\vec{X}^{\star}=\vec{X}-\vec{L}$, which describes the motion in the plane, and to note that $\dot{\vec{X}}=\dot{\vec{X}}^{\star}$.

The dynamics implied by Eqs. (66) and (76) is quite counterintuitive and it is useful to first develop some feeling for its content. One of the outstanding features of these equations of motion is the appearance of a limiting speed. One of the obvious questions is, what happens if an object is accelerated up to this limiting speed? To develop some understanding of this, we focus on the long-scale approximation (76). Let us therefore consider these equations of motion in the presence of a constant outward radial force, i.e., a potential of the form $-\beta R, \beta>0$. As a benchmark, we first integrate the equations of motion (59)-(62) with this potential. We take $\beta=5$ and as initial conditions $z_{1}=$ $\bar{z}_{1}=w_{1}=\bar{w}_{1}=1$ and compute $w_{1}$ and $\bar{w}_{1}$ from the constraint for physicality of the wave function in Eq. (63). For the coordinates, this choice corresponds to the initial conditions $\vec{X}=\{2,0,0\}$ and $\vec{V}=\{0,0,0\}$. The results are shown in Figs. 1(a) and 1(b).

The surprise is that the motion is not simply a constant outward radial acceleration as one would naively expect. The projectile accelerates until it reaches the limiting speed $V=1$, and then it starts to decelerate. When its speed vanishes, its radial motion reverses and it continues accelerating radially inwards until it reaches the limiting speed, after which it again starts to decelerate until its speed vanishes and the cycle is repeated. Exactly the same result is obtained by integrating Eq. (76), but in this case one has to switch between the branches at the turning points in the speed (the change in sign of the acceleration is related to the flip in sign between the two branches). To understand the origin of this oscillatory motion, we return to the conserved energy and compute the effective potential for the radial motion. We therefore set $W(R)=-\beta R$ (we ignore the noncommutative correction to the potential here): (a)

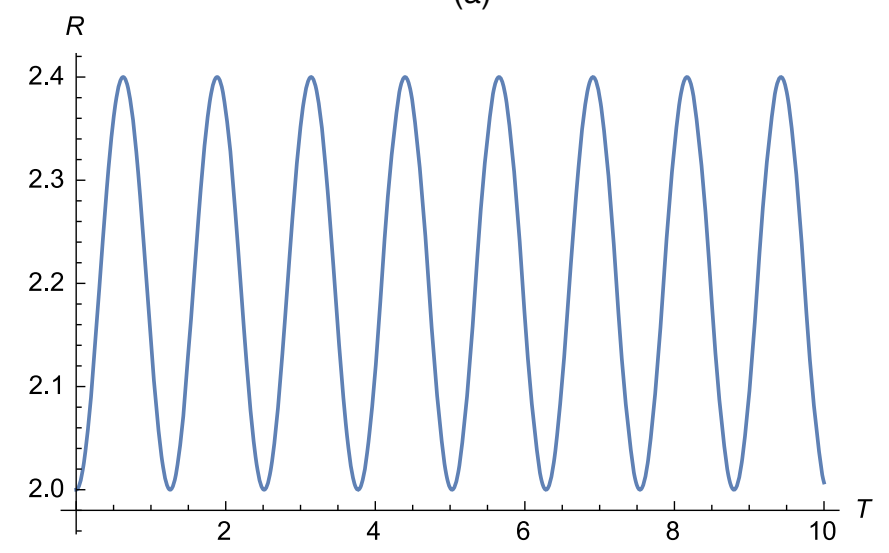

(b)

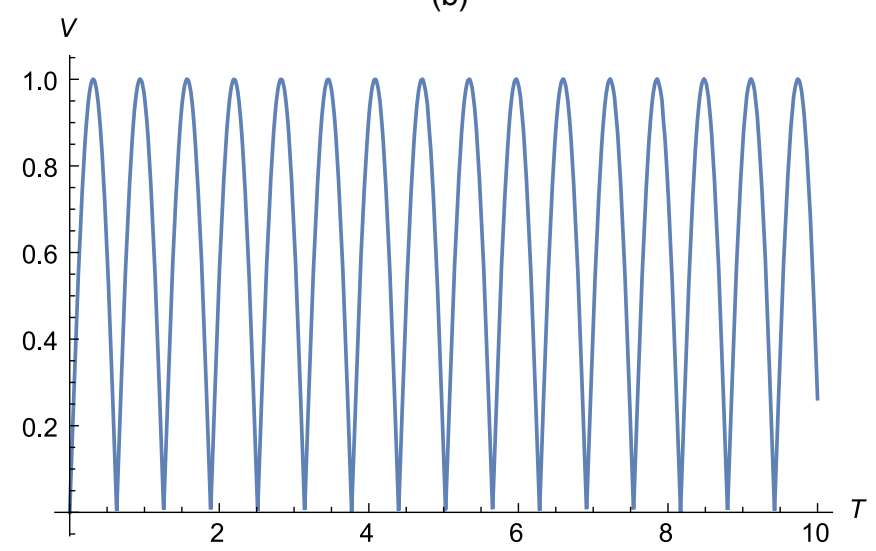

FIG. 1. Radius (a) and speed (b) of a particle subjected to a constant force in the outward radial direction. 


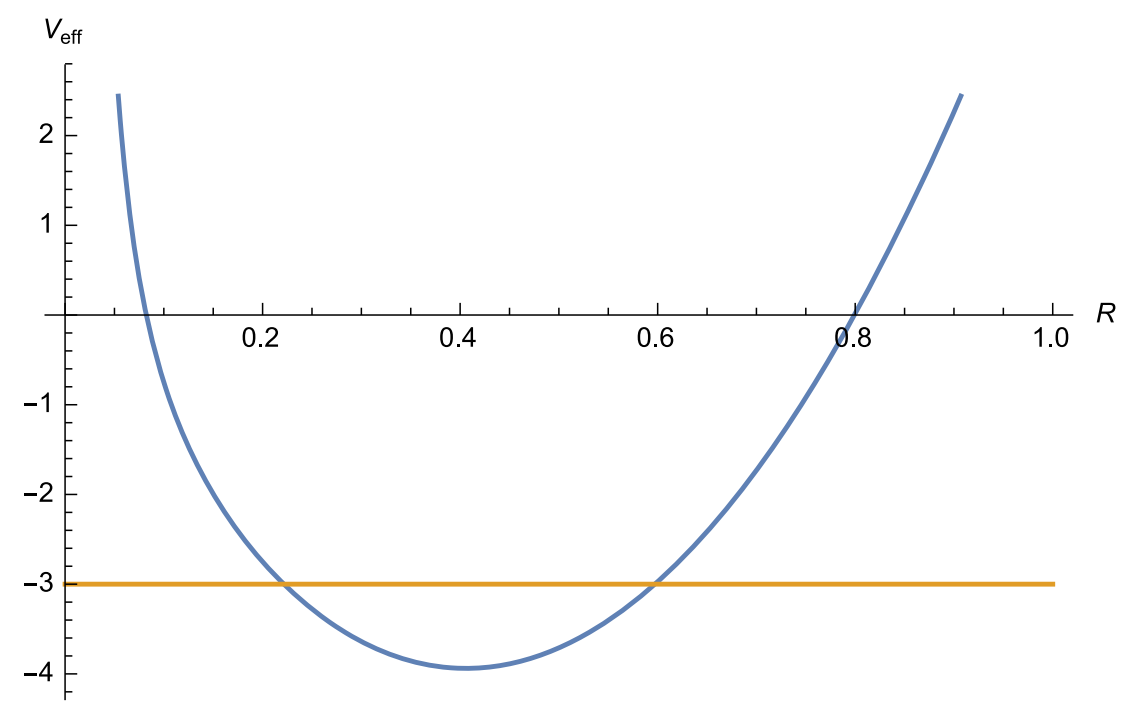

FIG. 2. Effective potential for the radial motion in the presence of a radially outwards constant force. In this figure $\beta=5, E=-1$, and $L=0.1$.

$$
E_{ \pm}=1 \pm \sqrt{1-\dot{\vec{X}} \cdot \dot{\vec{X}}}-\beta R
$$

Using $\dot{\vec{X}}^{2}=\dot{R}^{2}+\frac{L^{2}}{R^{2}}$, which can easily be checked from Eq. (77), this can be rewritten as

$\dot{R}^{2}+2(E-1) \beta R+\beta^{2} R^{2}+\frac{L^{2}}{R^{2}} \equiv \dot{R}^{2}+V_{\text {eff }}=E(2-E)$.

We observe that this is indeed a harmonic oscillator potential with a shifted minimum and a repulsive barrier at the origin. Note that for $E>2$, the left-hand side is positive but the right-hand side is negative, and this equation cannot be satisfied for these energies. This again demonstrates the cutoff in energy at $E=2$ referred to earlier.

In the case above, $L=0$ and the repulsive barrier is absent. In general, for $L \neq 0$ it is present, and the generic effective potential is shown in Fig. 2 with $\beta=5, E=-1$, and $L=0.1$. Also shown is the right-hand side of Eq. (86) (horizontal line). The points where the horizontal line intersects the curve of $V_{\text {eff }}$ are the turning points of the radial motion, as $\dot{R}=0$ at these points. The origin of the oscillatory motion seen in Fig. 1 should now be clear. Also note that the analysis in terms of the effective potential is independent of the branch. The message to take away from this exercise is that the square-root-based dispersion relation in Eq. (78), which is also the source of the limiting speed, can give rise to rather peculiar and counterintuitive dynamical behavior. However, when reformulated in terms of an effective potential, the dynamical behavior becomes very transparent.

We now turn to the case of gravity, for which the dimensionless potential reads

$$
W(R)=-\frac{\beta}{R}, \quad \beta=\frac{G M m^{2} \lambda}{\hbar^{2}}-1 .
$$

For our present purposes it is again sufficient to consider only the long-length-scale behavior where we can approximate the functions $f_{i}(R)$ as in Eq. (75). We again construct the effective potential, which now reads

$\dot{R}^{2}+\frac{2(E-1) \beta}{R}+\frac{\beta^{2}+L^{2}}{R^{2}} \equiv \dot{R}^{2}+V_{\mathrm{eff}}=E(2-E)$.

First, note that the $1 / R$ term in the effective potential (88) switches sign between $E<1$ and $E>1$ and that the effective potential is strictly repulsive for $E>1$. This is again a manifestation of the two branches already mentioned. Second, note that for $E>2$, the left-hand side of Eq. (88) is strictly positive, while the right-hand side is negative, resulting in the energy cutoff $E<2$ observed before. Third, note that there is always a repulsive barrier, even when $L=0$ on the short to medium length scales. This is quite different to the commutative case where the centrifugal term stabilizes the orbits. It should, however, be kept in mind that the behavior of the effective potential at short length scales may be drastically altered by the shortlength-scale corrections to the functions $f_{i}(R)$.

Since $\dot{\vec{X}}=\dot{\vec{X}}^{\star}$, the points where $E(2-E)-V_{\text {eff }}$ vanishes are the turning points of the orbitals where $\dot{R}=\dot{R}^{\star}=0$ [see also Eq. (96)]. These are the points where the line $E(2-E)$ intersects the curve of $V_{\text {eff }}$ in Fig. 3. This is shown in Figs. 3(a), 3(b), and 3(c) for $E<0,0<E<1$, and $1<E<2$, respectively. When $E<0$ as shown in Fig. 3(a), there are two turning points, where $R$ reaches its maximum and minimum. The motion is elliptic and the turning points represent the closest and furthest points of the orbit. When $0<E<1$ as in Fig. 3(b), there is only one 

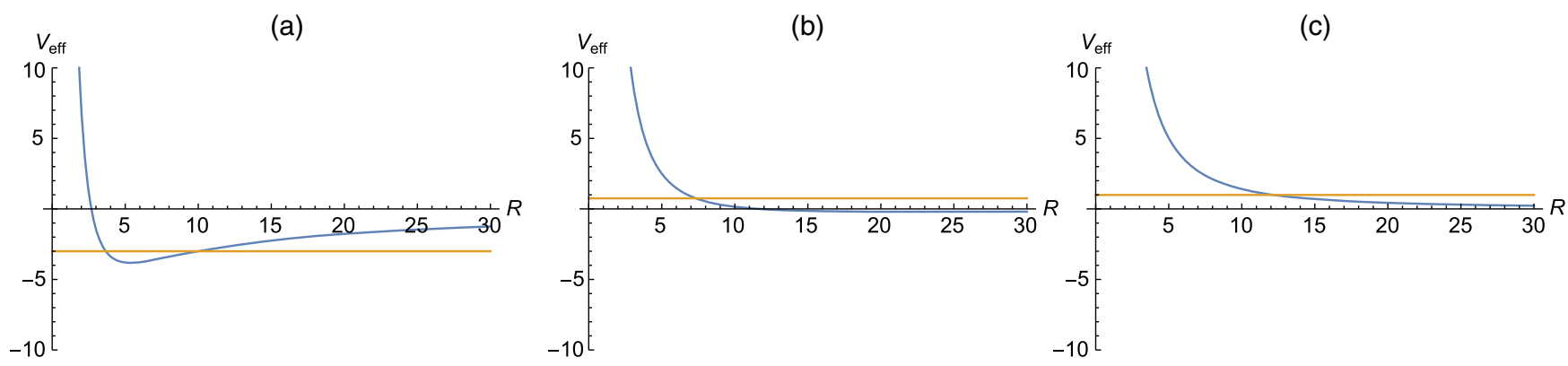

FIG. 3. Turning points of the orbitals for (a) $E<0(E=-0.5)$, (b) $0<E<1$ ( $E=0.5$ ), and (c) $1<E<2$ ( $E=1.1$ ). The horizontal lines are the values of $E(2-E) \cdot \beta=10$ and $L=0.1$ in these plots.

turning point, despite the fact that the potential still has an attractive tail. The particle is unbound and escapes to infinity. When $1<E<2$ as in Fig. 3(c), the potential is repulsive and a particle placed anywhere accelerates to infinity. Note, though, that its energy and speed is bounded by 2 and 1 , respectively. When the turning points coincide, which only happens at the minimum of the potential, the motion is circular. For this to happen $E$ and $L$ must be related in a specific way.

\section{B. Precession in a gravitational potential}

One expects that the modified dynamics implied by the noncommutativity can cause precession of elliptic orbitals, and we investigate this possibility here. It turns out that this is only possible if the short-length-scale corrections in the functions $f_{i}(R)$ are included. Since we are interested in bound orbitals, we take $E<0$ from here on.

We start by deriving the general expression for the precession angle. Without loss of generality, we can choose $\vec{L}$ along the $z$ direction and for our present purpose it is also convenient to restore dimensions from here on. Let us introduce the vector $\vec{x}^{\star}=\vec{x}-\frac{\lambda}{\hbar} \vec{e}$. From Eq. (84) it has the property $\vec{\ell} \cdot \vec{x}^{\star}=0$ and thus represents the rotating vector in the plane of motion. This vector only depends on the azimuthal angle $\phi$ and not on $\theta$, as is the case with $\vec{x}$. To establish precession, we must therefore compute the dependence of this vector on $\phi$.

To do this, we note from Eq. (84) and our choice of $\vec{\ell}$ along the $z$ axis $\left(\hat{\ell}\right.$ denotes the unit vector and $\left.\ell^{2}=\vec{\ell} \cdot \vec{\ell}\right)$ that

$$
\hat{\ell} \cdot \vec{x}=r \cos \theta=\frac{\lambda}{\hbar} \ell .
$$

Introducing $\vec{x} \cdot \vec{x}=r^{2}$ and $\vec{x}^{\star} \cdot \vec{x}^{\star}=r^{\star 2}$, we also have

$$
r^{2}=r^{\star 2}+\frac{\lambda^{2}}{\hbar^{2}} \ell^{2}
$$

From this we obtain

$$
\begin{aligned}
& \cos \theta=\frac{\lambda \ell}{\hbar \sqrt{r^{\star 2}+\frac{\lambda^{2} \ell^{2}}{\hbar^{2}}}}, \\
& \sin \theta=\frac{r^{\star}}{\hbar \sqrt{r^{\star 2}+\frac{\lambda^{2} \ell^{2}}{\hbar^{2}}}} .
\end{aligned}
$$

Differentiating the second of these with respect to time gives

$$
\dot{\theta}=\frac{\lambda \ell \dot{r}^{\star}}{\hbar \sqrt{r^{\star 2}+\frac{\lambda^{2} \ell^{2}}{\hbar^{2}}}} .
$$

Combining this with

$$
\ell^{2}=\frac{m^{2} \lambda^{2} r^{2}}{4 f_{2}(r / \lambda)^{2}}\left(\dot{\theta}^{2}+\sin ^{2} \theta \dot{\phi}^{2}\right)
$$

gives an equation for $\dot{\phi}$ :

$$
\dot{\phi}=\frac{2 f_{2}(r / \lambda) \ell}{r^{\star} m \lambda} \sqrt{1-\frac{m^{2} \lambda^{4}\left(\dot{r}^{\star}\right)^{2}}{4 f_{2}(r / \lambda)^{2} \hbar^{2}\left(r^{\star 2}+\frac{\lambda^{2} \ell^{2}}{\hbar^{2}}\right)}} .
$$

The final step is to eliminate $\dot{r}^{\star}$ from this equation. For this we use Eq. (70) to write

$$
\begin{aligned}
\dot{r}^{2}= & \frac{4 f_{2}(r / \lambda)^{2} r^{2} \hbar^{2}}{m^{2} \lambda^{4}}-\left(\frac{\lambda}{\hbar}\right)^{2} \\
& \times\left(e-\frac{\hbar^{2} r}{m \lambda^{3}}\left(f_{1}(r / \lambda)+f_{3}(r / \lambda)\right)-w(r)\right)^{2} \\
& -\frac{4 f_{2}(r / \lambda)^{2} \ell^{2}}{m^{2} \lambda^{2}} \equiv \Delta(r) .
\end{aligned}
$$

Using

$$
\dot{r}=\frac{\dot{r}^{\star} r^{\star}}{\sqrt{r^{\star 2}+\frac{\lambda^{2} \ell^{2}}{\hbar^{2}}}}
$$

and Eq. (90), we can write 


$$
\dot{r}^{\star}=\frac{\sqrt{\left(r^{\star 2}+\frac{\lambda^{2} \ell^{2}}{\hbar^{2}}\right) \Delta\left(r^{\star}\right)}}{r^{\star}},
$$

with $\Delta\left(r^{\star}\right) \equiv \Delta\left(\sqrt{r^{\star 2}+\frac{\lambda^{2} \ell^{2}}{\hbar^{2}}}\right)$. Substituting this into Eq. (94) gives

$$
\dot{\phi}=\frac{2 f_{2}\left(r^{\star} / \lambda\right) \ell}{r^{\star} m \lambda} \sqrt{1-\frac{m^{2} \lambda^{4} \Delta\left(r^{\star}\right)}{4 f_{2}\left(r^{\star} / \lambda\right)^{2} r^{\star 2} \hbar^{2}}} .
$$

Here $f_{2}\left(r^{\star} / \lambda\right)=f_{2}\left(\frac{1}{\lambda} \sqrt{r^{\star 2}+\frac{\lambda^{2} \ell^{2}}{\hbar^{2}}}\right)$. From this the precession angle for half of a cycle is easily obtained as

$$
\begin{aligned}
\Delta \phi= & \int_{r_{-}^{\star}}^{r_{+}^{\star}} d r^{\star} \frac{2 f_{2}\left(r^{\star} / \lambda\right) \ell}{m \lambda \sqrt{\left(r^{\star 2}+\frac{\lambda^{2} \ell^{2}}{\hbar^{2}}\right) \Delta\left(r^{\star}\right)}} \\
& \times \sqrt{1-\frac{m^{2} \lambda^{4} \Delta\left(r^{\star}\right)}{4 f_{2}\left(r^{\star} / \lambda\right)^{2} r^{\star 2} \hbar^{2}}} .
\end{aligned}
$$

Here $r_{ \pm}^{\star}$ are the turning points.

This form is still inconvenient as it is difficult to solve for the energy and angular momentum in terms of the turning points $r_{ \pm}^{\star}$. It is much easier to solve for them in terms of the turning points $r_{ \pm}$. We therefore make a change of variables in the integral back to these quantities by using Eq. (90). This yields

$$
\begin{aligned}
\Delta \phi= & \int_{r_{-}}^{r_{+}} d r \frac{2 f_{2}(r / \lambda) \ell}{m \lambda \sqrt{\left(r^{2}-\frac{\lambda^{2} \ell^{2}}{\hbar^{2}}\right) \Delta(r)}} \\
& \times \sqrt{1-\frac{m^{2} \lambda^{4} \Delta(r)}{4 f_{2}(r / \lambda)^{2} \hbar^{2}\left(r^{2}-\frac{\lambda^{2} \ell^{2}}{\hbar^{2}}\right)}} .
\end{aligned}
$$

The way we proceed is as follows. We choose turning points $r_{+}$and $r_{-}$and solve for the energy $e$ and angular momentum $\ell$ from the conditions [see Eq. (95)]

$$
\Delta\left(r_{+}\right)=\Delta\left(r_{-}\right)=0 .
$$

There are two pairs of solutions, but the one pair has positive energy and complex angular momentum and is therefore unphysical. The second pair has negative energy and real angular momentum and therefore describes elliptic motion. The two solutions in this pair are simply related by a change of sign of the angular momentum. We do not list these expressions explicitly due to their length.

The resulting integral (100) cannot be performed exactly, but we can attempt an expansion of the integrand in orders of $\lambda$ and integrate term by term. We do this for general functions $f_{i}(R)$, but it turns out to be convenient to write these functions in the following way:

$$
f_{i}(R)=\frac{1}{R} h_{i}\left(\frac{1}{R}\right)
$$

The only restriction at this point on the functions $h_{i}(x)$ is that $h_{i}(0)=\frac{1}{2}$, which ensures the desired asymptotic behavior as reflected in Eq. (75). In the special case of Eq. (74), it is easy to read off the explicit forms of these functions. This long calculation yields for the precession over half of a cycle

$$
\Delta \phi= \pm\left(\pi+\frac{\pi G M\left(r_{-}+r_{+}\right)\left(h_{1}^{\prime \prime}(0)-2 h_{2}^{\prime \prime}(0)+h_{3}^{\prime \prime}(0)\right)}{4 r_{-} r_{+}\left(1+h_{1}^{\prime}(0)-2 h_{2}^{\prime}(0)+h_{3}^{\prime}(0)\right)^{2}}\left(\frac{\lambda m}{\hbar}\right)^{2}+O\left(\lambda, \hbar^{0}\right)+O\left(\lambda^{2}, \hbar^{0}\right)\right)
$$

The sign depends on the choice of solutions, i.e., positive or negative angular momentum, which in turn depends on whether $1+h_{1}^{\prime}(0)-2 h_{2}^{\prime}(0)+h_{3}^{\prime}(0)$ is positive or negative. For convenience we only consider the positive case from here on. We note that if we take the limit $\lambda \rightarrow 0$ with $\hbar$ fixed, we recover the Newtonian result $\Delta \phi=\pi$. Also note that we cannot take the $\hbar \rightarrow 0$ limit before the $\lambda \rightarrow 0$ limit. However, if we take the $\lambda \rightarrow 0$ and $\hbar \rightarrow 0$ limits such that $\frac{\lambda}{\hbar}$ is a fixed ratio, i.e.,

$$
\frac{\lambda}{\hbar}=\frac{1}{m v_{0}},
$$

where $v_{0}$ is the limiting speed of the noncommutative system, the terms $O\left(\lambda, \hbar^{0}\right)+O\left(\lambda^{2}, \hbar^{0}\right)$ vanish and we get
$\Delta \phi=\pi+\frac{\pi G M\left(r_{-}+r_{+}\right)\left(h_{1}^{\prime \prime}(0)-2 h_{2}^{\prime \prime}(0)+h_{3}^{\prime \prime}(0)\right)}{4 r_{-} r_{+} v_{0}^{2}\left(1+h_{1}^{\prime}(0)-2 h_{2}^{\prime}(0)+h_{3}^{\prime}(0)\right)^{2}}$.

Introducing the length of the semimajor axis $a=\left(r_{+}+r_{-}\right) / 2$ and the eccentricity $\epsilon=\frac{r_{+}-r_{-}}{r_{+}+r_{-}}$, this reads

$\Delta \phi=\pi+\frac{\pi G M\left(h_{1}^{\prime \prime}(0)-2 h_{2}^{\prime \prime}(0)+h_{3}^{\prime \prime}(0)\right)}{2 a\left(1-\epsilon^{2}\right) v_{0}^{2}\left(1+h_{1}^{\prime}(0)-2 h_{2}^{\prime}(0)+h_{3}^{\prime}(0)\right)^{2}}$.

Substituting the form of the functions $h_{i}(R)$ as extracted from Eq. (74), one obtains 


$$
\Delta \phi=\pi+\frac{\pi G M}{8 a\left(1-\epsilon^{2}\right) v_{0}^{2}}
$$

Note that since Eq. (106) depends at most on the secondorder derivatives of $h_{i}(R)$, the inclusion of higher-order terms for $f_{2}$ in Eq. (74) cannot alter the result. Remarkably, this result has the same form as the general-relativistic (GR) result [18]

$$
\Delta \phi=\pi+\frac{3 \pi G M}{c^{2} a\left(1-\epsilon^{2}\right)},
$$

except for a numerical factor and the appearance of the limiting speed, rather then the speed of light. We discuss the physical ramifications of this result in Sec. VI.

\section{Stable circular orbitals in a gravitational potential}

In this section we study the behavior of stable circular orbits. We make the following ansatz for these orbitals:

$$
\begin{aligned}
& x(t)=r \sin \theta \cos (\omega t), \quad y(t)=r \sin \theta \sin (\omega t), \\
& z(t)=r \cos \theta .
\end{aligned}
$$

The only time dependence is therefore in the azimuthal angle $\phi$ that changes at a constant rate.

This ansatz is inserted into the equations of motion (66) for the negative branch and with a gravitational potential as in Eq. (87). We first consider the equation of motion in the $z$ direction, from which one can solve for $\cot \theta$ in terms of the speed $V^{2}=\dot{\vec{X}} \cdot \dot{\vec{X}}$ as

$$
\cot \theta=\frac{V}{\sqrt{4 R^{2} f_{2}(R)^{2}-V^{2}}} .
$$

Using this result in the equation of motion for the $x$ and $y$ directions, which collapse to the same equation, one obtains the velocity as

$$
V=4 R f_{2}(R) \sqrt{\frac{a b}{2(a b+c+d \sqrt{e})}},
$$

where

$$
\begin{aligned}
a= & \beta+R^{2}\left(R\left(f_{1}^{\prime}(R)-2 f_{2}^{\prime}(R)+f_{3}^{\prime}(R)\right)+f_{1}(R)-2 f_{2}(R)+f_{3}(R)\right), \\
b= & \beta+R^{2}\left(R\left(f_{1}^{\prime}(R)+2 f_{2}^{\prime}(R)+f_{3}^{\prime}(R)\right)+f_{1}(R)+2 f_{2}(R)+f_{3}(R)\right), \\
c= & 4 R^{4}\left(f_{2}(R)^{2}-R^{2} f_{2}^{\prime}(R)^{2}\right), \\
d= & \beta+R^{2}\left(R\left(f_{1}^{\prime}(R)+f_{3}^{\prime}(R)\right)+f_{1}(R)+f_{3}(R)\right), \\
e= & \beta^{2}+R^{2}\left(R\left(2 f_{3}^{\prime}(R)\left(\beta+R^{3} f_{1}^{\prime}(R)\right)+R^{3} f_{1}^{\prime}(R)^{2}+2 \beta f_{1}^{\prime}(R)-16 R^{2} f_{2}(R) f_{2}^{\prime}(R)+R^{3} f_{3}^{\prime}(R)^{2}\right)\right. \\
& +2 f_{3}(R)\left(\beta+R^{3}\left(f_{1}^{\prime}(R)+f_{3}^{\prime}(R)\right)\right)+2 f_{1}(R)\left(\beta+R^{2}\left(R\left(f_{1}^{\prime}(R)+f_{3}^{\prime}(R)\right)\right.\right. \\
& \left.\left.\left.+f_{3}(R)\right)\right)+R^{2} f_{1}(R)^{2}+R^{2} f_{3}(R)^{2}\right) .
\end{aligned}
$$

Substituting Eq. (111) into Eq. (110) gives $\cot \theta$ as a function of radius.

These equations do not provide much insight into the behavior of the velocity and $\cot \theta$ as a function of radius. To simplify matters, we consider the long-length-scale behavior in which we approximate the functions $f_{i}(R)$ as in Eq. (75). After restoring dimensions using Eq. (51) and setting $\beta=\frac{G M m^{2} \lambda}{\hbar^{2}}-1 \approx \frac{r_{0}}{\lambda}$ with $r_{0}=\frac{G M}{v_{0}^{2}}$, this gives

$$
\begin{aligned}
& v(r)=v_{0} \sqrt{\frac{2}{1+\sqrt{1+4\left(\frac{r}{r_{0}}\right)^{2}}}} \\
& \cot \theta=\sqrt{\frac{2}{\sqrt{1+4\left(\frac{r}{r_{0}}\right)^{2}}-1}}
\end{aligned}
$$

We note the following interesting behavior:

$$
\begin{aligned}
& v(r)=v_{0}, \quad r \ll r_{0}, \\
& v(r)=\sqrt{\frac{G M}{r},} \quad r \gg r_{0} .
\end{aligned}
$$

Note, however, that the constant behavior does not extend down to small radii as the short-length-scale corrections to the functions $f_{i}(R)$, which we neglected, become important and at short lengths scales one must consider the full expression (111). This result is intuitively simple to understand. If there is a bounding speed, the dependence of the velocity on radius must be modified at small distances to avoid a violation of this limiting speed. The only question is at what length scale this modification takes effect. We leave the discussion of the physical implications for Sec. VI. 


\section{GENERALIZED DYNAMICS}

In this section we consider a generalization of the results in the previous sections, based on the possible modification of the functions $f_{i}$ that appear in Eqs. (55)-(57). The source of such a modification of the functions $f_{i}$ relates to the choice of inner product on the quantum Hilbert space. In this regard it is crucial to realize that the choice of inner product and Laplacian is intimately connected by the requirement of Hermiticity of the Laplacian. Indeed, it can easily be checked that Eq. (37) is Hermitian with respect to Eq. (34). This choice of the inner product and Laplacian in turn determines the form of the functions $f_{i}(R)$ recorded in Eqs. (55)-(57). If one changes the inner product from Eq. (34) to a more general form,

$(\psi \mid \phi)=4 \pi \lambda^{3} \operatorname{tr}_{c}\left(\psi^{\dagger} f^{2}(\hat{R} / \lambda) \phi\right) \equiv 4 \pi \lambda^{3} \operatorname{tr}_{c}\left(\psi^{\dagger} f^{2}(\hat{n}+1) \phi\right)$,

for some nonvanishing, real function $f$, the Laplacian (37) also needs to be changed to

$$
\begin{aligned}
\hat{\Delta}|\psi\rangle & \left.=-\mid \frac{1}{\lambda^{2} f^{2}(\hat{R} / \lambda)}\left[\hat{a}_{\alpha}^{\dagger},\left[\hat{a}_{\alpha}, \psi\right]\right]\right) \\
& \left.=\mid \frac{1}{\lambda^{2} f^{2}(\hat{n}+1)}\left[\hat{a}_{\alpha}^{\dagger},\left[\hat{a}_{\alpha}, \psi\right]\right]\right)
\end{aligned}
$$

in order to maintain Hermiticity. By doing this one can quickly retrace the steps leading to the functions $f_{i}(R)$ given in Eqs. (55)-(57) to find that the modified functions are then

$$
\begin{gathered}
f_{1}(R)=\frac{1}{2}\left\langle z_{\alpha}\left|\frac{1}{f^{2}(\hat{n}+2)}\right| z_{\alpha}\right\rangle, \\
f_{2}(R)=\frac{1}{2}\left\langle z_{\alpha}\left|\frac{1}{f(\hat{n}+1) f(\hat{n}+2)}\right| z_{\alpha}\right\rangle, \\
f_{3}(R)=\frac{1}{2}\left\langle z_{\alpha}\left|\frac{1}{f^{2}(\hat{n}+1)}\right| z_{\alpha}\right\rangle .
\end{gathered}
$$

Note that Eq. (34) corresponds to the choice $f(x)=\sqrt{x}$. This modification has two consequences: 1) the trace of the operator that projects onto the subspace of spheres with radius $r \leq \lambda(\hat{n}+1)$ no longer yields the volume of a sphere in Euclidean space, and 2) the dispersion relation of the free-particle Schrödinger equation is modified. Although this is acceptable at short length scales, these modifications are unwanted at long length scales and we therefore require that $f(x)$ has the asymptotic behavior $f(x) \rightarrow \sqrt{x}$ when $x \rightarrow \infty$. This generalization may therefore be interpreted as introducing some form of curvature on configuration space, but such that it is asymptotically flat. This provides a paradigm for a generalized interpretation of the equations of motion (67) and constants of motion (69) where the functions $f_{i}(R)$ are treated as generalized functions as in Eqs. (117)-(119). Note that these functions are not completely arbitrary, but rather are determined by one single function $f(x)$.

\section{DISCUSSION}

We have now collected the most important results following from the noncommutative classical dynamics on fuzzy space. The challenge that remains is to extract a coherent physical scenario from these unconventional results. We discuss each result separately. Our discussion assumes a gravitational potential, which is the most relevant from an observational point of view.

\section{A. Limiting energy and speed}

One of the central features, which has cropped up on several occasions in the discussion above, is the existence of a cutoff energy with value $\frac{2 \hbar^{2}}{m \lambda^{2}}$, even for a free particle. This result was also found in earlier studies of quantum mechanics on fuzzy space where it essentially appears because the de Broglie wavelength cannot be made smaller than the noncommutative length scale. The existence of an energy cutoff is certainly reasonable from the point of view of gravitational stability and one may hope that it may help to regulate ultraviolet divergences in a full-fledged field theory.

Another striking result is the existence of a limiting speed $v_{0}=\frac{\hbar}{m \lambda}$. The existence of such a limiting speed in itself is not so unconventional as we know that the speed of light also presents such a limit, but rather its dependence on the mass of the projectile creates interpretational difficulties. In the case of gravity the minus branch of the equation of motion (79), which reduces to the Newtonian limit, reads

$$
\ddot{\vec{x}}=\frac{G M}{r^{3}}\left[\frac{m \lambda}{\hbar}(\vec{x} \times \dot{\vec{x}})-\sqrt{1-\left(\frac{m \lambda}{\hbar}\right)^{2} \dot{\vec{x}} \cdot \dot{\vec{x}} \vec{x}}\right] .
$$

If we assume that $\lambda$ is some fixed parameter only determined by the properties of space, this seemingly contradicts one of the most established principles in physics that motion under a gravitational force is independent of the mass, or indeed any other properties of the projectile, which is clearly not the case for Eq. (120). This is also the foundation for the geometrical interpretation of gravity as developed by Einstein and has been experimentally verified to great accuracy by the Dicke-Eötvös experiment.

There are two possible ways out of this dilemma. The first point of view is that the noncommutative parameter is so small and, correspondingly, the limiting speed so high that the dependence on the mass of the projectile is undetectable. This certainly requires the limiting speed to be much greater than the speed of light to avoid any observational conflict. This point of view is certainly also 
not out of step with current thinking on general relativity and quantum gravity, which anticipates a possible modification in particle dynamics at short length scales. However, this point of view also presents some difficulties. If one believes that Eq. (120) can be applied to macroscopic and astrophysical objects, which have very large masses, it should be clear that the noncommutative parameter is severely restricted to very small values, generally much less than even the Planck length, to be compatible with the motion observed for astrophysical objects. Indeed, a simple order-of-magnitude estimate shows that the noncommutative parameter must be less than $10^{-62} \mathrm{~m}$ to avoid the limiting speed of Earth to be less than the observed speed relative to the Sun. If one takes the noncommutative parameter to be a universal constant, this effectively forces a commutative scenario with very small possibility of observing any noncommutative effects.

One may object to the application of Eq. (120) on macroscopic or astrophysical scales, arguing that these are massive, extended objects composed of a huge number of microscopic particles. The key element to keep in mind here is that the basic entity on which we build both the quantum and classical descriptions of a system is the action. On the microscopic level the action feeds into the path integral to compute quantum-mechanical transition amplitudes, and on macroscopic scales the requirement that the action is stationery provides us with the equations of motion. Here, just as in the commutative case, we have the action and consistency requires us to follow the same philosophy in both cases. In the commutative case we freely apply Newton's equations on macroscopic and astrophysical scales and it would certainly seem irrational not to do the same in the case of Eq. (120). The rationale behind this extrapolation to astrophysical scales is that the size of astrophysical objects is still very small compared to solar or galactic length scales and that a point-particle approximation is therefore reasonable. This approximation is even made in the relativistic case. Of course, we know that size does matter and that the finite sizes of the Sun and Mercury give rise to orbital precession effects that have to be disentangled from the purely relativistic, or anomalous, precession. In spirit, the application of Eq. (120) on solar and galactic scales is the same.

Although the point of view presented above may very well be true, it is worthwhile to explore alternatives. A second point of view one can take to avoid the dilemma posed by Eq. (120) is to assume that the limiting speed

$$
\frac{\hbar}{m \lambda}=v_{0}
$$

is a universal constant, independent from any properties of the projectile. This requires us to adopt the point of view that the commutation relations of the coordinates of a macroscopic particle with mass $m$ are given by

$$
\left[\hat{x}_{i}, \hat{x}_{j}\right]=\frac{2 \hbar}{m v_{0}} i \varepsilon_{i j k} \hat{x}_{k} .
$$

This implies that the properties of noncommutative space, or at least the coordinates of a massive particle moving in noncommutative space, must depend on the mass of the projectile, i.e., the noncommutative parameter must undergo some form of renormalization due to the presence of the projectile. This is not a completely foreign notion as we know from GR that the local properties of space-time will be modified by the presence of a projectile.

One may be concerned that noncommutative effects may now get out of control for microscopic objects. However, what one must keep in mind is that Eq. (120) and the argument leading to Eq. (122) lose their validity in this case. In this regard it is important to realize that Eq. (120) is only valid when both quantum fluctuations (controlled by $\frac{\hbar}{m}$ ) and coordinate fluctuations (controlled by the noncommutative parameter) are small. It is also for this reason that the order of limits $\hbar \rightarrow 0$ and $\lambda \rightarrow 0$ is important. Taking the $\hbar \rightarrow 0$ limit while keeping $\lambda$ fixed leads to nonsensical results since the coordinate fluctuations remain relevant. As the coordinate fluctuations are in this case controlled by the momentum scale $m v_{0}$, Eqs. (120) and (122) are only valid when this momentum scale is large enough. Note that in the $\hbar \rightarrow 0$ limit these fluctuations automatically vanish. Noncommutative effects can therefore not get out of control, unless one applies these equations outside their range of validity. Indeed, when a proper quantum-mechanical treatment is done as in Refs. [14,16], complete consistency with standard quantum mechanics is found. Note that Eqs. (120) and (122) remain valid for massive projectiles even for small limiting speeds.

It is at this point not clear what the value of $v_{0}$ should be. It is, of course, tempting to adopt the speed of light as its value, but there are no compelling arguments for this as the equations of motion derived here only apply to massive particles and have nothing to say about the propagation of light. The latter has to concern itself with the formulation of Maxwell's equations on fuzzy space.

If one considers the result (107) for the precession and if this must represent a small correction to the relativistic anomalous precession (108), one concludes that $v_{0} \gg c$, at least on solar scales, as already argued above. Note that in the limit $v_{0} \rightarrow \infty$, one recovers the commutative scenario with standard Newtonian dynamics. This scenario would therefore represent a small perturbation to Newtonian dynamics, which, depending on the value of $v_{0}$, may be completely undetectable with current observational techniques. In this scenario any potential observational conflict can therefore be avoided. Of course, in this scenario the standard problems associated with the Newtonian paradigm, such as the velocity curves of galaxies, are still present. 


\section{B. Velocity curves}

One of the most attractive features of the current dynamics is the flatness of the velocity curve below the length scale $r_{0}=\frac{G M}{v_{0}^{2}}$. From Eq. (114) we also note that the plateau value of the speed is precisely $v_{0}$. Clearly, in the scenario above where $v_{0} \gg c$ the plateau value is too high and the length scale $r_{0}$ too short to have any observational consequences. The above scenario certainly seems to be forced on us on solar scales, but if one adopts the point of view that the limiting speed may have a spatial dependence, possibly as described in Sec. V, one may argue for much lower limiting speeds on galactic scales that may have clear observational consequences.

Although there are no compelling empirical or theoretical arguments for this assumption, let us assume the existence of a possibly spatial-dependent limiting speed much less than the speed of light (of the order of $100-300 \mathrm{~km} \mathrm{~s}^{-1}$ ) in the noncommutative scenario described above. If one moves out from the center of a galaxy, the velocity curve grows simply because the included mass grows and several models for this exist [19]. However, once the velocity reaches the limiting velocity, the curve must saturate at this value and stay there up to the length scale $r_{0}$, after which it will assume the standard $\sqrt{\frac{G M}{r}}$ behavior as in Eq. (114). This does, of course, require us to take the limiting velocity as the plateau velocity, commonly denoted $v_{f}$, of the observed velocity curve. The important point to realize though is that in this scenario flat velocity curves are natural and indeed generic and no specific distribution of the mass in the galaxy has to be assumed for flatness. In fact, one may assume that all of the mass is concentrated at the center, as we indeed did when deriving Eq. (113).

Figure 4 shows data for the Milky Way from Ref. [20] with galactic constants $R_{0}=8 \mathrm{kpc}$ and $V_{0}=200 \mathrm{~km} \mathrm{~s}^{-1}$

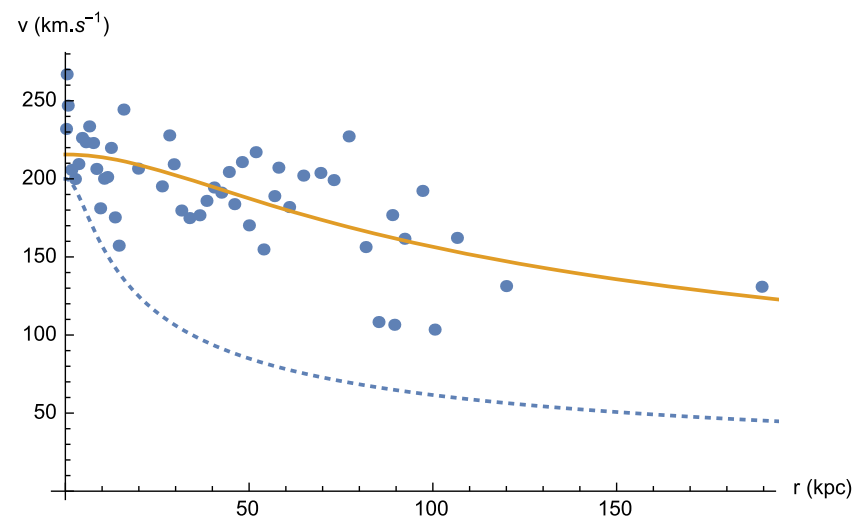

FIG. 4. Velocity curve and data for the Milky Way. The data is from Ref. [20] with galactic constants $R_{0}=8 \mathrm{kpc}$ and $V_{0}=200 \mathrm{~km} \mathrm{~s}^{-1}$. The dashed curve is for an estimated baryonic mass of $9.3 \times 10^{10} M_{\odot}$ and $v_{0}=200 \mathrm{~km} \mathrm{~s}^{-1}$, which gives $r_{0}=9.96 \mathrm{kpc}$. The solid line is a least-squares fit of the velocity curve (113) to the data with mass $8.3 \times 10^{11} M_{\odot}$ and $v_{f}=v_{0}=215.6 \mathrm{~km} \mathrm{~s}^{-1}$. up to $200 \mathrm{kpc}$. We also show a least-squares fit of the velocity curve (113) to the data (solid line). This gives $r_{0}=$ $76.5 \mathrm{kpc}$ and $v_{0}=215.6 \mathrm{~km} \mathrm{~s}^{-1}$, which requires a galactic mass of $8.3 \times 10^{11} M_{\odot}$. This is in complete agreement with the mass $M(200 \mathrm{kpc})=6.8 \pm 4.1 \times 10^{11} M_{\odot}$ reported in Ref. [20], but larger then the mass $M(100 \mathrm{kpc})=3 \times$ $10^{11} M_{\odot}$ reported in Ref. [19]. Note, however, the difference in radius so that a larger value is to be expected. Let us also consider what happens if we just consider the baryonic mass (stellar and gaseous). A reliable estimate of this can be obtained from the empirical baryonic Tully-Fisher relation [21]

$$
a_{0} G M=v_{f}^{4} .
$$

We take for the constant $a_{0}$ the value reported in Ref. [21] of $a_{0}=1.3 \times 10^{-10} \mathrm{~m} \mathrm{~s}^{-2}$ and $v_{f}=200 \mathrm{~km} \mathrm{~s}^{-1}$. Using these values we find the baryonic mass of the Milky Way to be $9.3 \times 10^{10} M_{\odot}$. This gives $r_{0}=9.96 \mathrm{kpc}$ for $v_{0}=v_{f}=$ $200 \mathrm{~km} \mathrm{~s}^{-1}$. This velocity curve is also shown as the dotted line in Fig. 4. Clearly, the value of $r_{0}$ is too small if only baryonic mass is considered to explain the extent of the plateau observed in the velocity curve. Indeed, as usual, we see that the baryonic mass only makes up around $11 \%$ of the galactic mass required to explain the data. However, as mentioned before, in this scenario no assumptions about the distribution of this excess mass in the galaxy needs to be made to explain the flatness of the velocity curve. It may therefore even be possible that this mass is concentrated in the center of the galaxy, e.g., in the form of a massive black hole. One may be concerned that this concentration of mass may be detectable through the motion of nearby stars such as S2 [22], and this would certainly be the case in a Newtonian paradigm. However, in the current paradigm the limiting speed may prevent such a detection if it is low enough and the application of a Newtonian paradigm will lead to an underestimation of the mass. In fact, in the current paradigm the only way that the included mass can be estimated accurately is through the length scale $r_{0}$ as the velocity is largely independent from the included mass below this scale. This does, however, pose a further difficulty. The speed of S2 at its perihelion is around $5000 \mathrm{~km} \mathrm{~s}^{-1}$ [22], much larger than the plateau value of the velocity curve of around $200 \mathrm{~km} \mathrm{~s}^{-1}$. If all of the excess mass is concentrated at the center of the galaxy, it requires us to assume that the limiting velocity must be spatially dependent, which can be accommodated through a generalized function $f_{2}(r)$ as described in Sec. V.

Finally, note that if we accept, as argued above, that $v_{0} \gg c$ on solar scales, the standard Newtonian paradigm holds and no observational conflict with the velocity curves on solar scales can result.

\section{Two branches}

One of the features of the equations of motion derived above is the existence of two branches with disconnected 
dynamics, of which only one reduces to standard Newtonian dynamics in the commutative limit. The choice of branch is determined by the value of the energy as reflected in Eq. (80) and, since energy is conserved, is set by the initial conditions. In particular, we note that for $\frac{\hbar^{2}}{m \lambda^{2}}<e<\frac{2 \hbar^{2}}{m \lambda^{2}}$ or, equivalently, $\frac{\hbar v_{0}}{\lambda}<e<\frac{2 \hbar v_{0}}{\lambda}$, the dynamics must be described by the plus branch, which does not reduce to Newtonian dynamics in the commutative limit. Note, however, from Eq. (80) that high velocities are not required for this energy range. On the unified level of the effective potential for the radial motion discussed in Sec. IVA, the presence of these two branches manifests itself in that the effective potential becomes completely repulsive for the range of energies (88). This implies that a projectile with energy in this range will indefinitely accelerate radially outwards.

\section{Displaced planar motion}

A feature that sets the current dynamics apart from Newtonian or general-relativistic dynamics is the offset of the source of the gravitational potential from the plane of motion. This is encoded in Eq. (89) where the offset of the angle $\theta$ from $\frac{\pi}{2}$ measures this displacement. One can, of course, also express it in terms of the absolute distance $r \cos \theta$ appearing in Eq. (89). Assuming the asymptotic form (75) for the functions $f_{i}$, one can easily check that the magnitude of the angular momentum in Eq. (89) coincides with the commutative result, i.e., $\vec{\ell}=m(\vec{x} \times \dot{\vec{x}})$. Note, though, that this is only true for the magnitude and not the individual components of the commutative angular momentum, which are in fact not even conserved. We have a simple result for the magnitude of the commutative angular momentum in terms of the parameters of the elliptic orbit of the projectile:

$$
\ell=m \sqrt{\frac{2 G M r_{+} r_{-}}{r_{+}+r_{-}} .}
$$

Using this in Eq. (89), we obtain for the displacement $d$, which is also a constant of motion,

$$
d=r \cos \theta=\frac{1}{v_{0}} \sqrt{\frac{2 G M r_{+} r_{-}}{r_{+}+r_{-}} .}
$$

Hence, this displacement gives a direct measure of the limiting speed $v_{0}=\frac{\hbar}{m \lambda}$ of the projectile.

We note that if a measurement of this offset gives a zero value, this supports a commutative scenario or at least a scenario in which $v_{0}$ is large. If it yields a nonzero value, this supports a noncommutative scenario. If this offset can be measured for projectiles with different masses, one may also be able to distinguish between the mass-independent or mass-dependent noncommutative scenarios discussed in Sec. VI A.

\section{SUMMARY AND CONCLUSIONS}

We have derived the path-integral action for a particle moving in fuzzy space and the corresponding classical equations of motion. The main features of these equations are a cutoff energy, a generally spatial-dependent limiting speed, planetary precession remarkably similar to the general-relativistic result, velocity curves that plateau below the length scale $\frac{G M}{v_{0}^{2}}$, displaced planar motion, and the existence of two dynamical branches of which only one reduces to Newtonian dynamics in the commutative limit. The branch that does not reduce to Newtonian dynamics predicts a repulsive effective potential for the radial motion and indefinite outward acceleration. Most of these features are unconventional and observational data pose a severe challenge for this scenario. Given that it is not easy to write down symmetry-preserving noncommutative scenarios for space-time, this may even present a challenge to any such scenario.

We have also unpacked several physical scenarios that are compatible with the above results. In particular, a scenario with a universal limiting speed $v_{0} \gg c$ gives a very standard paradigm that can avoid any possible observational conflict. Of course, this scenario then also does not offer any solutions to the problems associated with the standard paradigm.

An interesting scenario that has not been explored here is the possibility that relativistic effects may emerge out of a noncommutative scenario. The emergence of a limiting speed and the remarkable correspondence between the noncommutative and relativistic orbital precessions certainly points in this direction.
[1] N. Seiberg, arXiv:hep-th/0601234.

[2] H. S. Snyder, Phys. Rev. 71, 38 (1947).

[3] S. Doplicher, K. Fredenhagen, and J. E. Roberts, Commun. Math. Phys. 172, 187 (1995).

[4] N. Seiberg and E. Witten, J. High Energy Phys. 09 (1999) 032.
[5] F. G. Scholtz, L. Gouba, A. Hafver, and C. M. Rohwer, J. Phys. A 42, 175303 (2009).

[6] M. R. Douglas and N. A. Nekrasov, Rev. Mod. Phys. 73, 977 (2001).

[7] F. G. Scholtz and J. Govaerts, J. Phys. A 41, 505003 (2008).

[8] J. N. Kriel and F. G. Scholtz, J. Phys. A 45, 095301 (2012). 
[9] F. G. Scholtz, J. N. Kriel, and H. W. Groenewald, Phys. Rev. D 92, 125013 (2015).

[10] T. Cliftona, P. G. Ferreiraa, A. Padillab, and C. Skordisb, Phys. Rep. 513, 1 (2012).

[11] S. Nojiri and S. D. Odintsov, Int. J. Geom. Methods Mod. Phys. 04, 115 (2007).

[12] J. Klauder and B. Skagerstam, Coherent States: Applications in Physics and Mathematical Physics (World Scientific, Singapore, 1985).

[13] S. Gangopadhyay and F. G. Scholtz, Phys. Rev. Lett. 102, 241602 (2009).

[14] N. Chandra, H. W. Groenewald, J. N. Kriel, F. G Scholtz, and S. Vaidya, J. Phys. A 47, 445203 (2014).
[15] J. N. Kriel, H. W. Groenewald, and F. G. Scholtz, Phys. Rev. D 95, 025003 (2017).

[16] V. Gáliková and P. Prešnajder, J. Math. Phys. (N.Y.) 54, 052102 (2013).

[17] H. C. Steinacker, Nucl. Phys. B910, 346 (2016).

[18] J. B. Hartle, Gravity (Addison Wesley, San Francisco, 2003).

[19] Y. Sofue, M. Honma, and T. Omodaka, Publ. Astron. Soc. Jpn. 61, 227 (2009).

[20] P. Bhattacharjee, S Chaudhury, and S. Kundu, Astrophys. J. 785, 63 (2014).

[21] S. S. McGaugh, Astron. J. 143, 40 (2012).

[22] R. Schödel et al., Nature (London) 419, 694 (2002). 